\begin{tabular}{|c|c|}
\hline Title & $\begin{array}{l}\text { Development of an optical time-resolved measurement system under high-pressure and low-temperature with a piston- } \\
\text { cylinder pressure cell }\end{array}$ \\
\hline Author(s) & T suchiya, Satoshi; Kino, Y ohei; Nakagawa, Koichi; Nakagawa, Daisuke; Y amada, Jun-ichi; Toda, Y asunori \\
\hline Citation & $\begin{array}{l}\text { Review of Scientific Instruments, 87(4), } 043104 \\
\text { https://doi.org/10.1063/1.4945303 }\end{array}$ \\
\hline Issue Date & 2016 \\
\hline Doc URL & http:/hdl.handle.net/2115/61101 \\
\hline Rights & $\begin{array}{l}\text { Copyright } 2016 \text { A merican Institute of Phy sics. This article may be downloaded for personal use only. A ny other use } \\
\text { requires prior permission of the author and the A merican Institute of Physics. The following article appeared in Rev. } \\
\text { Sci. Instrum. 87, 043104 (2016) and may be found at } \\
\text { http://scitation.aip.org/content/aip/journal/ } / s i / 87 / 4 / 10.1063 / 1.4945303 .\end{array}$ \\
\hline Type & article \\
\hline File Information & 1.4945303.pdf \\
\hline
\end{tabular}

Instructions for use 


\section{AIP $\mid \begin{aligned} & \text { Review of } \\ & \text { Scientific Instruments }\end{aligned}$}

\section{Development of an optical time-resolved measurement system under high-pressure and low-temperature with a piston-cylinder pressure cell}

Satoshi Tsuchiya, Yohei Kino, Koichi Nakagawa, Daisuke Nakagawa, Jun-ichi Yamada, and Yasunori Toda

Citation: Review of Scientific Instruments 87, 043104 (2016); doi: 10.1063/1.4945303

View online: $\mathrm{http}: / / d x$. doi.org/10.1063/1.4945303

View Table of Contents: http://scitation.aip.org/content/aip/journal/rsi/87/4? ver=pdfcov

Published by the AIP Publishing

\section{Articles you may be interested in}

Pressure cell for transport measurements under high pressure and low temperature in pulsed magnetic fields

Rev. Sci. Instrum. 87, 023907 (2016); 10.1063/1.4941714

A compact bellows-driven diamond anvil cell for high-pressure, low-temperature magnetic measurements

Rev. Sci. Instrum. 85, 033901 (2014); 10.1063/1.4867078

Note: Compact optical fiber coupler for diamond anvil high pressure cells

Rev. Sci. Instrum. 84, 106108 (2013); 10.1063/1.4824486

Short piston-cylinder pressure cells based on $\mathrm{Ni}-\mathrm{Cr}-\mathrm{Al}$ cylinders and their application to fragile materials Rev. Sci. Instrum. 81, 033903 (2010); 10.1063/1.3310197

In situ pressure calibration for piston cylinder cells via ruby fluorescence with fiber optics

Rev. Sci. Instrum. 78, 066109 (2007); 10.1063/1.2749451

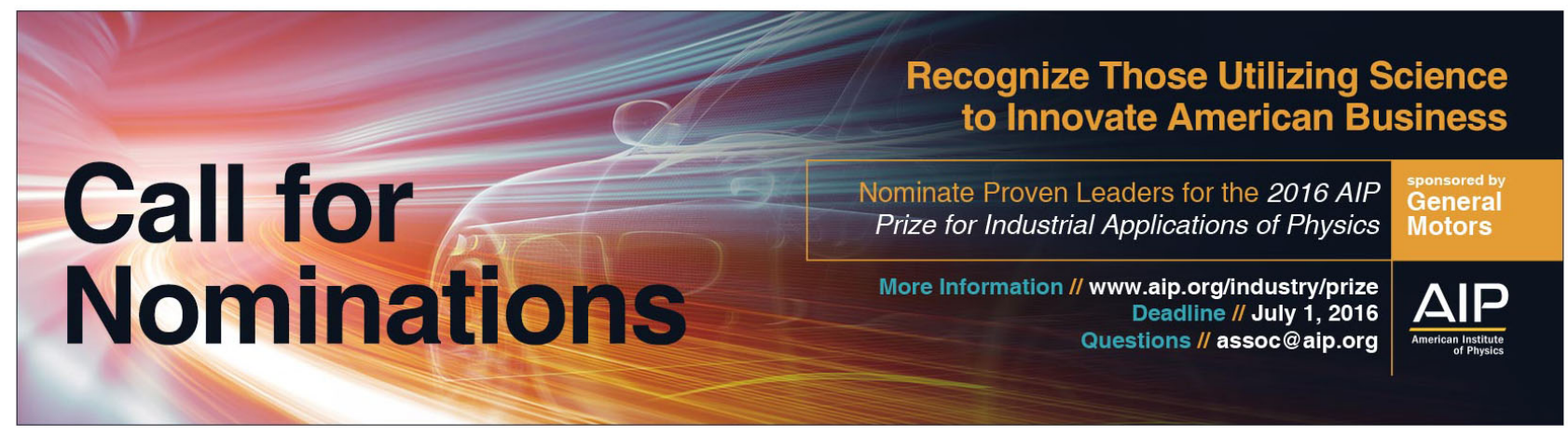




\title{
Development of an optical time-resolved measurement system under high-pressure and low-temperature with a piston-cylinder pressure cell
}

\author{
Satoshi Tsuchiya, ${ }^{1, a)}$ Yohei Kino, ${ }^{1}$ Koichi Nakagawa, ${ }^{1}$ Daisuke Nakagawa, ${ }^{1}$ \\ Jun-ichi Yamada, ${ }^{2}$ and Yasunori Toda ${ }^{1}$ \\ ${ }^{1}$ Department of Applied Physics, Hokkaido University, Hokkaido 060-8628, Japan \\ ${ }^{2}$ Graduate School of Material Science, University of Hyogo, Hyogo 650-004, Japan
}

(Received 20 November 2015; accepted 21 March 2016; published online 5 April 2016)

\begin{abstract}
To perform the femtosecond pump-probe spectroscopy under high pressure and low temperature, we constructed a measurement system with a piston cylinder type pressure cell installing an optical fiber bundle. The applied pressure was achieved to $6 \mathrm{kbar}$ and the cell was cooled down to $15 \mathrm{~K}$. Several demonstrations revealed that broadening and change of polarization of pulse (duration of $\sim 120 \mathrm{fs}$ ) owing to the dispersions in the fiber bundle are much small indicating that those have little influence on the measurement of carrier relaxation dynamics. In the measurements of $\kappa$-(BEDT-TTF $)_{2} \mathrm{Cu}(\mathrm{NCS})_{2}$ under $1.3 \mathrm{kbar}$ at $43 \mathrm{~K}$, we have successfully detected the polarization anisotropy of the carrier relaxation dynamics and estimated the decay time in the same way as the normal measurement. (C) 2016 AIP Publishing LLC. [http://dx.doi.org/10.1063/1.4945303]
\end{abstract}

\section{INTRODUCTION}

Femtosecond time-resolved optical spectroscopy is a powerful technique to measure relaxation of photo-excited quasi-particles (QPs) resolved in the time domain. This measurement has made a significant contribution to researches in a strongly correlated electron system such as cuprate and organic superconductors. ${ }^{1-21}$ Although, in this system, pressure and temperature are important parameters to control their electronic phases, systematic time-resolved measurements with varying pressure have not been performed so far. This is because the optical measurements under pressure tends to require large effort to prepare and handle a pressure-tunable equipment.

Under high pressure, various optical measurements have been performed by using a diamond anvil cell (DAC) because of optical transparency of diamond anvils. ${ }^{22,23}$ However, DAC tends to be expensive and difficult to handle because of small sample space (less than $10^{-3} \mathrm{~mm}^{3}$ ). Another equipment to achieve high pressure is a piston-cylinder type pressure cell. ${ }^{24}$ The piston-cylinder pressure cell has been widely used for electrical resistivity, magnetization, and thermal measurements and has larger sample space $\left(\sim 10^{2} \mathrm{~mm}^{3}\right)$ than that of DAC. To use the piston-cylinder cell for optical measurements, an optical window is needed to set in it. A sapphire and diamond were used as a window in the photoluminescence measurements ${ }^{25}$ and the infrared investigations under high pressure. ${ }^{26}$ On the other hand, an optical fiber is also suitable for the window since it enables light to propagate with little attenuation. Indeed, by building a thin fiber optics directly into the piston-cylinder cell, fluorescence of ruby has been measured. ${ }^{27}$ However, by use of the thin fiber, optical response from sample becomes often much small because the area irradiated by the beam is limited by the diameter of the fiber.

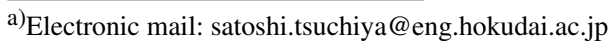

An optical fiber bundle which consists of a large number of the thin fibers can make it possible to carry out the optical measurements under pressure. Since the overall diameter of the bundle is sufficiently larger than sample, the response signal comes from the area limited by the spot size of the beam, which is in the same way as the measurement under ambient pressure. In this paper, we report the construction of femtosecond pump-probe measurement system with a pistoncylinder type pressure cell and a short optical fiber bundle. Through the several demonstrations, the following points were evaluated: attainable pressure and temperature, effect of the dispersions on an optical pulse, detection characteristics of transient change of reflectivity, and polarization in the organic conductor $\kappa$-(BEDT-TTF $)_{2} \mathrm{Cu}(\mathrm{NCS})_{2}$ (hereafter $\kappa$ NCS) under multiple conditions (43 K and $1.3 \mathrm{kbar}$ ).

\section{PRESSURE AND TEMPERATURE CHARACTERISTICS OF PRESSURE CELL}

To realize the time-resolved measurement under high pressure and low temperature, the piston-cylinder type hybrid pressure cell ${ }^{24}$ and optical fiber bundle were prepared. Our pressure cell was essentially the same with that in the previous report. ${ }^{24}$ Two-layered cylinder consists of a nonmagnetic Ni-Cr-Al and $\mathrm{Cu}-\mathrm{Be}$ alloys. Plug and clump bolts are $\mathrm{Cu}-\mathrm{Be}$. Piston and piston-back-up are made of a nonmagnetic tungsten carbide (WC). Figure 1(a) shows a schematic of a cross-sectional view of the sample space and microscopic image of the end surface of the fiber bundle. The plug has a hole whose diameter is $3.4 \mathrm{~mm}$ to set the optical fiber bundle in it. The fiber bundle consists of about 50 thousands narrow fibers (12 $\mu \mathrm{m}$ of diameter of the individual cores), whose overall diameter is $3.2 \mathrm{~mm}$ and length is $25.4 \mathrm{~mm}$ (the specifying part number: $\sharp 53-839$, Edmund Optics Inc.). Refractive index of core and cladding is 1.58 and 1.48, respectively. The bundle is set into the hole of plug and fixed tightly by stycast $(2850 \mathrm{FTJ})$. The stycast was poured into the space between the plug and fiber bundle to fill the 

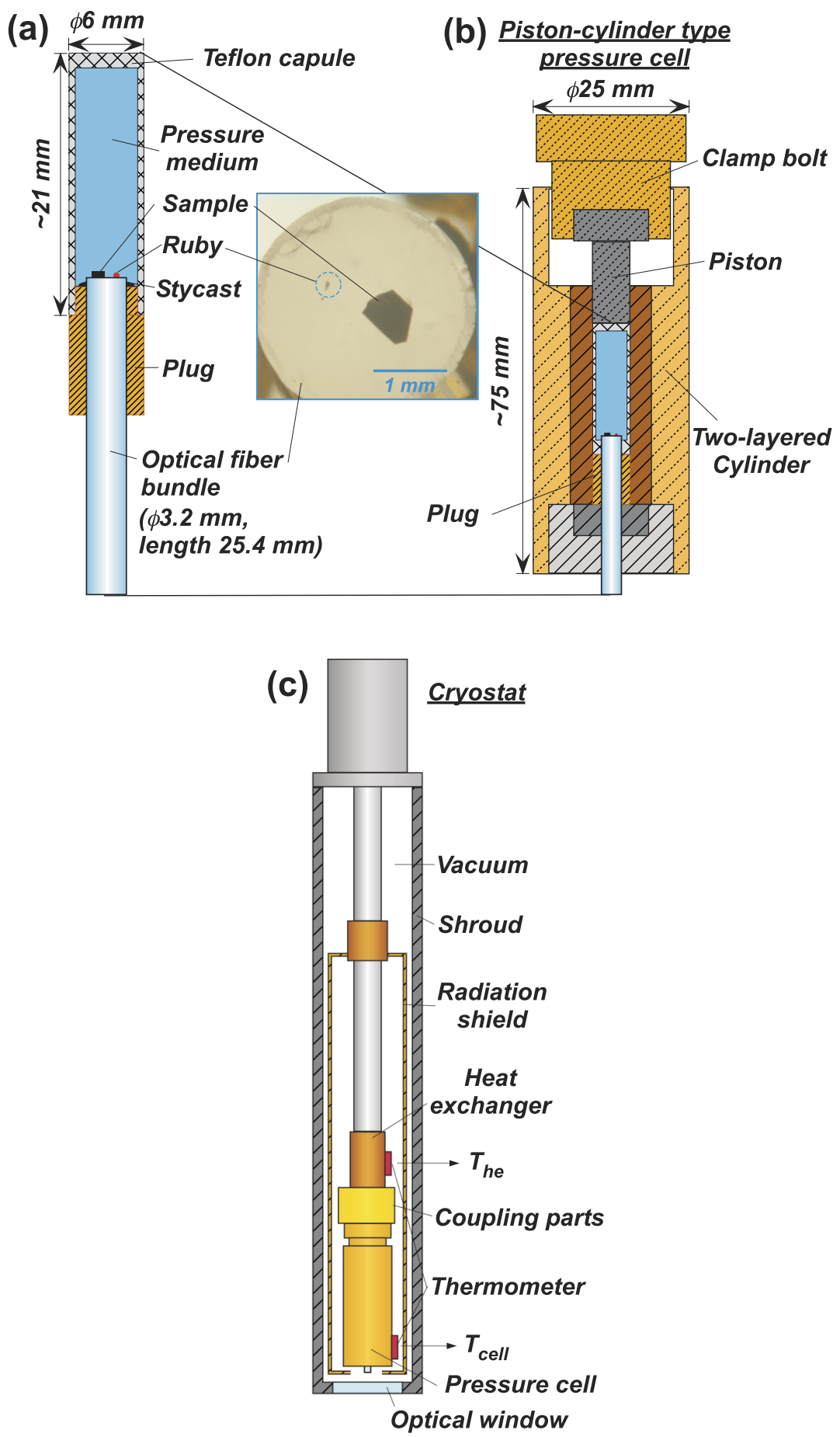

FIG. 1. (a) Cross-sectional view of the sample space and microscopic image of the end surface of optical fiber bundle. (b) Cross-sectional view of piston-cylinder type pressure cell. (c) Schematic of cryostat.

space as evenly as possible after the removal of dissolved gas under vacuum. Sample and ruby particles are put on the end surface of the fiber in the sample space. The plug was joined to Teflon capsule with the pressure medium (Daphne 7373) and mounted in the pressure cell as shown in Fig. 1(b). In this setup, a vent hole was not constructed at the space under the clamp bolt to release pressure in it. For safety, the hole would be better to be formed. Figure 1(c) shows cross-sectional view of the pressure cell, which is connected to heat exchanger of the cryostat with the brass joint and cooled with a helium flow.
Applied pressure was calibrated by the ruby fluorescence method. ${ }^{28}$ For excitation, green laser light $(532 \mathrm{~nm})$ was generated from the diode pumped solid state laser. The beam reflected by a dichroic mirror (DM) was focused by an objective lens and irradiated to ruby particles presented on the end of the bundle. The fluorescence comes through DM and into a charge coupled device spectrometer (Ocean Optics, HR4000) via an optical fiber.

The inset of Fig. 2 shows spectra of the ruby fluorescence for 0 and 2 ton. Both the $R_{1}$ and $R_{2}$ peaks are shifted 


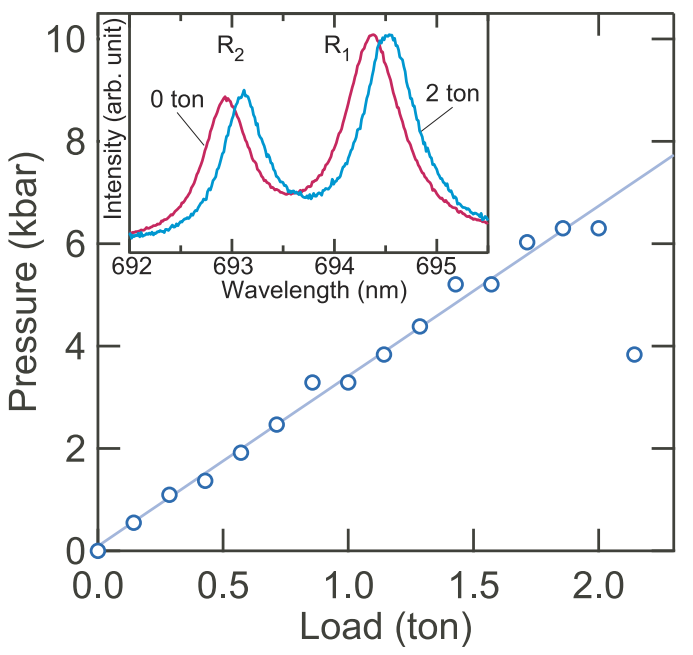

FIG. 2. The plot of pressure versus the load. The solid line indicates the linear fit. The inset shows ruby fluorescence spectra.

clearly to the long-wavelength side with increasing load. The applied pressure is estimated by the $R_{1}$ shift $\Delta R_{1}$ with the simple expression $P=2.740(\mathrm{kbar} / \AA) \times \Delta \mathrm{R}_{1}{ }^{28}$ The estimated pressure values are plotted as a function of the load in Fig. 2. The pressure was linearly applied up to $6 \mathrm{kbar}$ and suddenly reduced. This reduction was caused by disaggregation between the bundle and plug. An optimal size of the space between the bundle and plug remains unclear although it can be important to get high pressure. If the space is too large, the bundle can be fixed tilting with respect to the plug. This may cause breaking of the bundle. If the space is too small, amount of the stycast will reduce, leading to disaggregation between them. By improving condition of a diameter of the hole and adhesion between the bundle and plug, pressure is expected to increase more than $6 \mathrm{kbar}$.

Temperatures were measured by two thermometers which were attached to the end of the cell and exchanger as shown in Fig. 2(c). The cell was cooled down to $T_{\text {cell }} \sim 15 \mathrm{~K}$ and $T_{\text {he }}$ $\sim 4.2 \mathrm{~K}$, respectively, without any trouble.

\section{EXPERIMENTAL SETUP OF TIME-RESOLVED SPECTROSCOPY}

In the time-resolved spectroscopy, two optical pulses, namely, pump and probe pulses, are used. First pump pulse excites carriers in a solid to non-equilibrium high-energy states. The excited QPs relax immediately to states near the Fermi surface by electron-electron and electron-phonon $(e-p h)$ scattering. Second probe pulse measures the change of reflectivity $\Delta R / R$, which is associated with the nonequilibrium distribution of QPs as a function of delay time $t$. In the experiment, to obtain information in terms of the symmetry change in a system, we measured the probe polarization dependence. ${ }^{29,30}$ The $120 \mathrm{fs}$ pump and probe pulses centered at $400 \mathrm{~nm}$ and $800 \mathrm{~nm}$, respectively, are generated from a cavitydumped Ti:sapphire oscillator with repetition rates from 54 to $270 \mathrm{kHz}$ to avoid the heating effect. These beams were coaxially overlapped by DM and focused by an objective lens to sample presented on the end of the fiber bundle.
The positional relation between sample and the beams was monitored by image of CCD camera in real time and controlled precisely by translation of cryostat. The probe polarization was rotated by a half wave plate (HWP), where $\theta$ is the rotation angle of the probe electric field from the horizontal direction.

For a test sample, single crystals of $\kappa$-NCS were prepared. ${ }^{31}$ The conducting layer of BEDT-TTF molecules and insulating layer of $\mathrm{Cu}(\mathrm{NCS})_{2}$ are alternately stacked along the $a^{\star}$ axis, showing highly two-dimensional electronic nature. The crystals have plate-like shapes, whose sizes in the present experiments were $500 \times 200 \times 50 \mu^{3}$, as shown in Fig. 1(a). In the measurement, the optical pulses irradiated perpendicular to the conducting $(b-c)$ plane. The optical penetration depth is about $10 \mu \mathrm{m}$ and $3 \mu \mathrm{m}$ for pump and probe pulses, respectively ${ }^{32}$ indicating that the obtained data reflect a bulk property of $\kappa$-NCS. Under ambient pressure, $\kappa$-NCS shows the characteristic polarization anisotropy for the probe in the transient response below $70 \mathrm{~K}$, which can be related with glasslike structural transition. ${ }^{33}$ This response will be a benchmark for the demonstrations at low temperatures.

\section{PROPERTIES OF OPTICAL PULSE PASSING THROUGH A FIBER BUNDLE}

When an ultrashort optical pulse propagates in a fiber optics, broadening of the pulse, which will lead to reduction of time resolution on the measurements, is caused by the dispersions. To investigate the broadening effect on the transient measurement, we measured transient change of reflectivity $\Delta R / R$ by using the fiber bundle at room temperature. Figure 3(a) shows $\Delta R / R$ normalized by the maximum value $\Delta R_{\max }$ of $\kappa$-NCS obtained from the measurement with the fiber bundle and the normal measurement. Decay times $\tau$ were estimated by fitting the data with a single exponential function $\exp \left(-\frac{t}{\tau}\right)$. The fits yield $\tau_{\mathrm{f}} \sim 0.88 \mathrm{ps}$ for the measurement with the bundle which is comparable with $\tau_{\mathrm{w}} \sim 0.89 \mathrm{ps}$ for that without the bundle. To check the influence of pulse broadening more precisely, we also compare the initial rise of $\Delta R / R$. For simplicity, the rising times $t_{\mathrm{f}}$ and $t_{\mathrm{w}}$ were defined as a time between $\frac{1}{2} \Delta R_{\max }$ and $\Delta R_{\max }$ as shown in Fig. 3(a). As a result, $t_{\mathrm{f}}$ was $\sim 0.25 \mathrm{ps}$, which agrees with the $t_{\mathrm{w}}$ value $\sim 0.23 \mathrm{ps}$ within experimental error. These results indicate that the pulse broadening has almost no effect on the relaxation measurements.

Here, we roughly estimated the broadening of the pulse caused by the material dispersion. By assuming Gaussian pulse and using an input pulse width of $120 \mathrm{fs}$ and the group velocity dispersion of $36 \mathrm{fs}^{2} / \mathrm{mm}$ on silica glass at $800 \mathrm{~nm}$, the width of pulse passed through fiber was estimated as $\sim 122 \mathrm{fs}$. Such a small broadening will be unmeasurable, which was consistent with the above results. We have to note that the spatial mode dispersion of the fiber bundle would also broaden the pulse width. In the case of much shorter femtosecond pulse, the broadening could be larger than the theoretical estimation.

To realize the Raman-like analysis on the QP dynamics, we employ the polarization-resolved measurement. ${ }^{29,30}$ Since polarization of the optical pulse can be modulated by the polarization mode dispersion, the degree of polarization (DOP) of the pulse propagated through the fiber was evaluated 


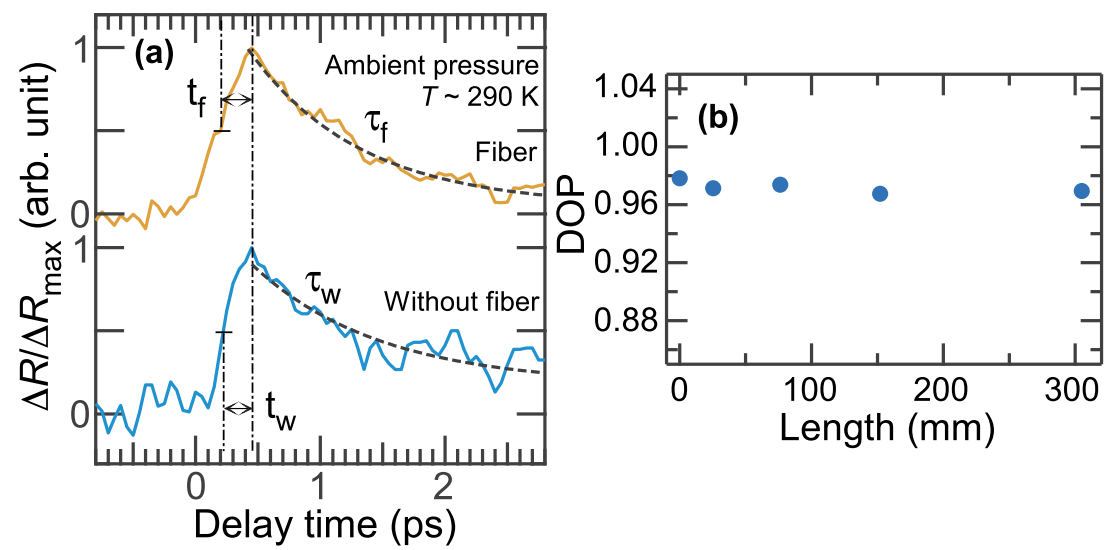

FIG. 3. (a) $\Delta R / R$ transients obtained from the measurement with the fiber optics for $F \sim 1100 \mu \mathrm{J} / \mathrm{cm}^{2}$ and the normal measurement under ambient pressure for $F=93 \mu \mathrm{J} / \mathrm{cm}^{2}$ at a room temperature. The dashed lines indicate the results fitted by a single exponential function. The data are normalized by the maximum values of $\Delta R$ and shifted for clarity. (b) Degree of polarization (DOP) as a function of length of the optical fiber bundle. The value at $0 \mathrm{~mm}$ corresponds to DOP of ambient light.

using Stokes parameters, which were obtained by combining a quarter wave plate and a polarizer. Theoretically, DOP has a value in the range from 0 to 1 . DOP $=0$ and 1 mean that light is unpolarized and completely polarized, respectively. Figure 3(b) shows the fiber-length dependence of DOP. The DOP value of ambient pulse presented at $0 \mathrm{~mm}$ is $\sim 0.978$, indicating that the pulse is mostly polarized. For the pulse propagated through the fiber bundle, the DOP values are nearly equal to those of the ambient pulse at any fiber-lengths. The results indicate that the polarization of pulse undergoes little change even with the use of the fiber bundle.

On the measurement using the fiber optics, power loss of pulses due to the non-linear effects caused by high-intensity light may be concerned. Since the non-linear effects depend on length of the fiber optics, we measured power $p_{b}$ of pulse passing through the bundle and compare it with $p$ which was measured before entering the bundle with four kind of bundles whose lengths are $25.4,76.2,152$, and $305 \mathrm{~mm}$. As a result,
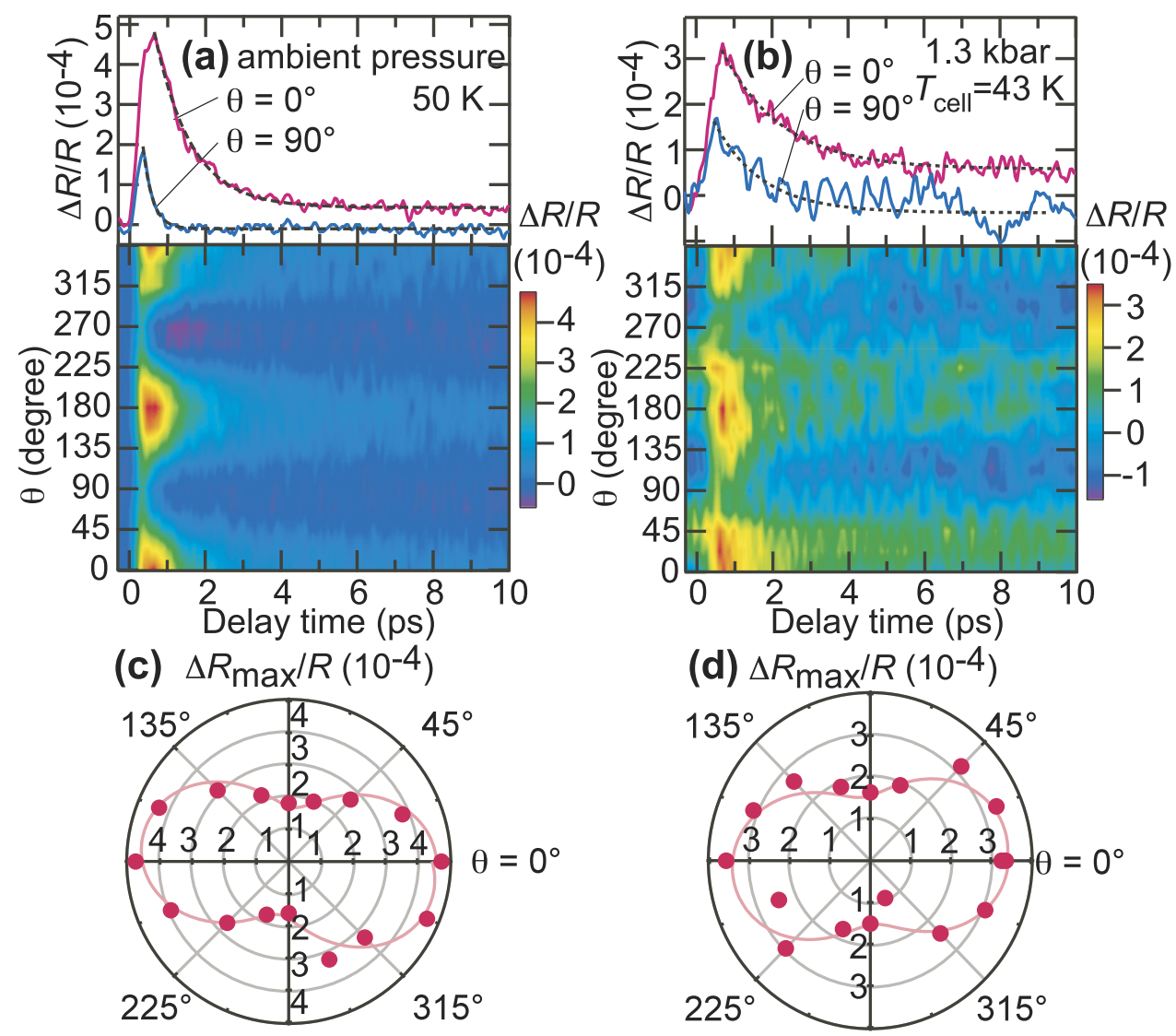

FIG. 4. ((a) and (b)) Transient changes of reflectivity $\Delta R / R$ for $\theta=0^{\circ}$ and $90^{\circ}$ and intensity plots of $\Delta R / R$ as a function of probe-polarization-angle under ambient pressure at $50 \mathrm{~K}$ for $F=121 \mu \mathrm{J} / \mathrm{cm}^{2}$ and $1.3 \mathrm{kbar}$ at $43 \mathrm{~K}$ for $F \sim 1300 \mu \mathrm{J} / \mathrm{cm}^{2}$, respectively. The dashed lines indicate the results fitted by a single exponential function. ((c) and (d)) Polar plots of the maximum values of $\Delta R / R$ under ambient pressure at $50 \mathrm{~K}$ and $1.3 \mathrm{kbar}$ at $43 \mathrm{~K}$, respectively. The solid lines indicate the results fitted by a double-angle function. 
for all the bundles, the ratios between them were $\frac{p_{b}}{p} \sim 0.4$, which corresponds to $35 \%-45 \%$ efficiency of transmission in the catalog. This means that the power loss was caused by reflection at an end face of the bundle. It is consistent with the fact that the laser power used in our experiments is less than or comparable to $\sim 0.5 \mathrm{~mW}$, which will be too small to cause the non-linear effects.

\section{DEMONSTRATION UNDER HIGH PRESSURE AND LOW TEMPERATURE}

Figure 4(a) shows transient change of reflectivity $\Delta R / R$ for probe polarization $\theta=0^{\circ}$ and $90^{\circ}$ and intensity plots of $\Delta R / R$ as a function of $\theta$ under ambient pressure at $50 \mathrm{~K}$. To obtain clear signal, the pressure measurements were carried out with higher pump fluence $F \sim 1300 \mu \mathrm{J} / \mathrm{cm}^{2}$ as compared to $121 \mu \mathrm{J} / \mathrm{cm}^{2}$ in the normal measurements. We found that $\Delta R / R$ clearly depended on the probe polarization angle. Figure 4(c) shows a polar plot of the maximum values of $\Delta R / R$. The signal was enhanced for $\theta \sim 0^{\circ}$ and $180^{\circ}$, which correspond to the directions along the $b$ axis. This polarization anisotropy can be attributed to spatial symmetry breaking due to the glass-like structural transition below $70 \mathrm{~K} .{ }^{33}$ Figures 4(b) and 4(d) show the polarization angular dependences of $\Delta R / R$ under $1.3 \mathrm{kbar}$ at $T_{\text {cell }}=43 \mathrm{~K}$, which were quite similar to those in the normal measurement. The slight difference of direction of the anisotropy between them can be due to the misalignment between the crystal axis and polarization of pulse. The conservation of polarization can be an important consequence for optical measurements in a pressure equipment. Generally, birefringence of the window can be caused by the stress of the high pressure, leading to non-conservation of polarization. Therefore, these results suggest that polarization is conserved even in our pressure equipment and the polarization properties of the QP relaxation dynamics are measurable in the same way as the normal measurement.

Although the polarization anisotropies are comparable, we found the difference between the decay times obtained from the pressure and normal measurements. Under $1.3 \mathrm{kbar}$, the decay times are $\sim 1.58$ and 1.03 ps for $\theta=0^{\circ}$ and $90^{\circ}$, respectively, while under ambient pressure, $\tau \sim 0.95$ and $0.25 \mathrm{ps}$, respectively. The difference of the pump fluence between the measurements does not influence the decay time above the saturation condition $\left(F_{\mathrm{th}} \sim 40 \mu \mathrm{J} / \mathrm{cm}^{2}\right)$. Generally, application of pressure modulates the $e$ - $p h$ interaction. Since the carrier relaxation rate $1 / \tau$ is proportional to the strength of the $e$-ph interaction, ${ }^{34,35}$ the applied pressure may lead to modulation of the decay time of the QP relaxation.

In the demonstrations, the pressure was calibrated at room temperature. In the previous report, ${ }^{36}$ the applied pressure is reduced for the Daphne 7373 pressure medium as temperature decreases, meaning that the pressure value at low temperatures can become smaller than 1.3 kbar. However, it is difficult to determine pressure accurately at low temperature by the ruby fluorescence method since the $\mathrm{R}_{1}$ and $\mathrm{R}_{2}$ lines of the ruby fluorescence are shifted depending on temperature. ${ }^{37}$ One of the solutions is that temperature dependence of the $R_{1}$ shift $\Delta R_{1}$ is investigated previously in the Daphne 7373 under $0 \mathrm{GPa}$. Because amount of $\Delta \mathrm{R}_{1}$ is almost unchanged even at low temperatures, ${ }^{38}$ a pressure value may be calibrated from the pre-investigations.

\section{SUMMARY}

We developed the measurement technique of femtosecond optical pump-probe spectroscopy under high-pressure and low-temperature by using the piston-cylinder type pressure cell and optical fiber bundle. The series of demonstrations revealed that our measurement system is available for the pump-probe measurement and provides reliable results in terms of the decay time and polarizations of the relaxation dynamics. The technique will be applicable to other optical measurements and become a key part of the time-resolved spectroscopy under multiple conditions including high magnetic field, opening a new research field.

\section{ACKNOWLEDGMENTS}

The authors would like to thank Y. Uwatoko, K. Matsubayashi, and S. Yamamoto for preparation of a piston-cylinder pressure cell, and K. Takemura and S. Klotz for support of the ruby pressure scale, and K. Yamane, K. Shigematsu, and T. Matsuura for valuable discussions and experimental supports. This work was partially supported by the Toyota Physical and Chemical Research Institute Scholars and a Grant-in-Aid for Young Scientists B (No. 15K17685) from the Japan Society for the Promotion of Science (JSPS).

${ }^{1}$ A. Rothwarf and B. N. Taylor, Phys. Rev. Lett. 19, 27 (1967).

${ }^{2}$ V. V. Kabanov, J. Demsar, B. Podobnik, and D. Mihailovic, Phys. Rev. B 59, 1497 (1999)

${ }^{3}$ J. Demsar, B. Podobnik, V. V. Kabanov, Th. Wolf, and D. Mihailovic, Phys. Rev. Lett. 82, 4918 (1999).

${ }^{4}$ R. A. Kaindl, M. Woerner, T. Elsaesser, D. Smith, J. Ryan, G. Farnan, M. McCurry, and D. Walmsley, Science 287, 470 (2000).

${ }^{5}$ R. D. Averitt, G. Rodriguez, A. I. Lobad, J. L. W. Siders, S. A. Trugman, and A. J. Taylor, Phys. Rev. B 63, 140502(R) (2001).

${ }^{6}$ D. Dvorsek, V. V. Kabanov, J. Demsar, S. M. Kazakov, J. Karpinski, and D. Mihailovic, Phys. Rev. B 66, 020510 (2002).

${ }^{7}$ Y. H. Liu, Y. Toda, K. Shimatake, N. Momono, M. Oda, and M. Ido, Phys. Rev. Lett. 101, 137003 (2008).

${ }^{8}$ P. Kusar, V. V. Kabanov, S. Sugai, J. Demsar, T. Mertelj, and D. Mihailovic, Phys. Rev. Lett. 101, 227001 (2008).

${ }^{9}$ T. Mertelj, V. V. Kabanov, C. Gadermaier, N. D. Zhigadlo, S. Katrych, J. Karpinski, and D. Mihailovic, Phys. Rev. Lett. 102, 117002 (2009).

${ }^{10}$ C. Gadermaier, A. S. Alexandrov, V. V. Kabanov, P. Kusar, T. Mertelj, X. Yao, C. Manzoni, D. Brida, G. Cerullo, and D. Mihailovic, Phys. Rev. Lett. 105, 257001 (2010).

${ }^{11}$ C. Giannetti, F. Cilento, S. Dal Conte, G. Coslovich, G. Ferrini, H. Molegraaf, M. Raichle, R. Liang, H. Eisaki, M. Greven, A. Damascelli, D. van der Marel, and F. Parmigiani, Nat. Commun. 2, 353 (2011).

${ }^{12}$ Y. Toda, T. Mertelj, P. Kusar, T. Kurosawa, M. Oda, M. Ido, and D. Mihailovic, Phys. Rev. B 84, 174516 (2011).

${ }^{13}$ L. Stojchevska, P. Kusar, T. Mertelj, V. V. Kabanov, Y. Toda, X. Yao, and D. Mihailovic, Phys. Rev. B 84, 180507(R) (2011).

${ }^{14}$ R. V. Yusupov, T. Mertelj, J. H. Chu, I. R. Fisher, and D. Mihailovic, Phys. Rev. Lett. 101, 246402 (2008).

${ }^{15}$ Y. Toda, R. Onozaki, M. Tsubota, K. Inagaki, and S. Tanda, Phys. Rev. B 80, 121103(R) (2009).

${ }^{16}$ T. Mertelj, P. Kusar, V. V. Kabanov, L. Stojchevska, N. D. Zhigadlo, S. Katrych, Z. Bukowski, J. Karpinski, S. Weyeneth, and D. Mihailovic, Phys. Rev. B 81, 224504 (2010).

${ }^{17}$ L. Stojchevska, P. Kusar, T. Mertelj, V. V. Kabanov, X. Lin, G. H. Cao, Z. A. Xu, and D. Mihailovic, Phys. Rev. B 82, 012505 (2010). 
${ }^{18}$ T. Naito, Y. Yamada, T. Inabe, and Y. Toda, J. Phys. Soc. Jpn. 77, 064709 (2008).

${ }^{19}$ T. Naito, M. Fukusaku, S. Matsuo, K. Tajima, T. Inabe, and Y. Toda, Physica B 405, S269 (2010).

${ }^{20}$ Y. Toda, T. Mertelj, T. Naito, and D. Mihailovic, Phys. Rev. Lett. 107, 227002 (2011).

${ }^{21}$ T. Naito, S. Matsuo, T. Inabe, and Y. Toda, J. Supercond. Novel Magn. 26, 1679 (2013).

${ }^{22}$ E. Eremets, High Pressure Experimental Method (Oxford University Press, New York, 1996)

${ }^{23}$ M. Kasami, T. Ogino, T. Mishina, S. Yamamoto, and J. Nakahara, J. Lumin. 119, 428 (2006).

${ }^{24}$ Y. Uwatoko, M. Hedo, N. Kurita, M. Koeda, M. Abliz, and T. Matsumoto, Physica B 329, 1658 (2003).

${ }^{25}$ J. D. Lambkin, B. J. Gunney, D. Lancefield, F. G. Bristrow, and D. J. Dunstan, J. Phys. E: Sci. Instrum. 21, 763 (1988).

${ }^{26}$ R. Beyer and M. Dressel, Rev. Sci. Instrum. 86, 053904 (2015).

${ }^{27}$ K. K. Nakazawa, M. Koeda, M. Hedo, and Y. Uwatoko, Rev. Sci. Instrum. 78, 066109 (2007).
${ }^{28}$ G. J. Piermarini, S. Block, J. D. Barnett, and R. A. Forman, J. Appl. Phys. 46, 2774 (1975).

${ }^{29}$ Y. Toda, F. Kawanokami, T. Kurosawa, M. Oda, I. Madan, T. Mertelj, V. V. Kabanov, and D. Mihailovic, Phys. Rev. B 90, 094513 (2014).

${ }^{30}$ S. Tsuchiya, Y. Sugawara, S. Tanda, and Y. Toda, J. Opt. 17, 085501 (2015).

${ }^{31}$ H. Anzai, J. M. Delrieu, S. Takasaki, S. Nakatsuji a, and J. Yamada, J. Cryst. Growth 154, 145 (1995).

${ }^{32}$ A. Ugawa, G. Ojima, K. Yakushi, and H. Kuroda, Phys. Rev. B 38, 5122 (1988).

${ }^{33}$ J. Muller, M. Lang, F. Steglich, J. A. Schlueter, A. M. Kini, and T. Sasaki, Phys. Rev. B 65, 144521 (2002).

${ }^{34}$ R. H. M. Groeneveld, R. Sprik, and A. Lagendijk, Phys. Rev. B 51, 11433 (1995).

${ }^{35}$ V. V. Kabanov and A. S. Alexandrov, Phys. Rev. B 78, 174514 (2008).

${ }^{36}$ K. Murata, H. Yoshino, H. O. Yadav, Y. Honda, and N. Shirakawa, Rev. Sci. Instrum. 68, 2490 (1997).

${ }^{37}$ D. D. Ragan, R. Gustavsen, and D. Schiferl, J. Appl. Phys. 72, 5539 (1992).

${ }^{38}$ K. Nakano, Y. Akahama, Y. Ohishi, and H. Kawamura, Jpn. J. Appl. Phys., Part 1 39, 1249 (2000). 